\title{
Chromosome specific control of chiasma frequency in rye
}

\author{
Julia White* and H. Rees
}

Department of Agricultural Botany, University College of Wales, Aberystwyth.

Genotypic control exercised by B chromosomes affects the mean chiasma frequency in pollen mother cells of rye. By using interchange to identify particular chromosomes it is established that B chromosomes affect chiasma formation in different chromosomes to varying degrees. That genotypic control is chromosome-specific signifies that variation in the amount of recombination may be adjusted independently among different gene complexes within the chromosome complement.

\section{INTRODUCTION}

There is plenty of evidence for genotypic control affecting the variation in the behaviour of chromosome complements at meiosis, particularly with regard to the frequency and localisation of chiasmata (Rees, 1957; Riley and Law, 1965). There are, however, few examples of control exercised upon specific chromosomes within the complements. The most notable are those described in Hypochoeris radicata by Parker (1975) and in Crepis capillaris by Tease and Jones (1976). The object of the present work was to seek further evidence of chromosome-specific control. This was done by comparing the response of particular chromosomes within the complement of rye to control exercised upon the chiasma frequency at meiosis by B chromosomes. That B chromosomes affect chromosome behaviour at meiosis in rye is well known (Jones and Rees, 1967). Particular chromosomes within the rye complement were "marked" in these experiments by means of structural rearrangement, namely interchange.

\section{MATERIALS AND METHODS}

\section{The interchange stock}

The interchange, designated $\mathrm{C}$, arose in a population derived from a cross between Secale cereale and $S$. vavilovii with B chromosomes, Both species are diploids with $2 n=14$. The interchange is

\footnotetext{
* Present address: Department of Biology, The University of Lancaster.
}

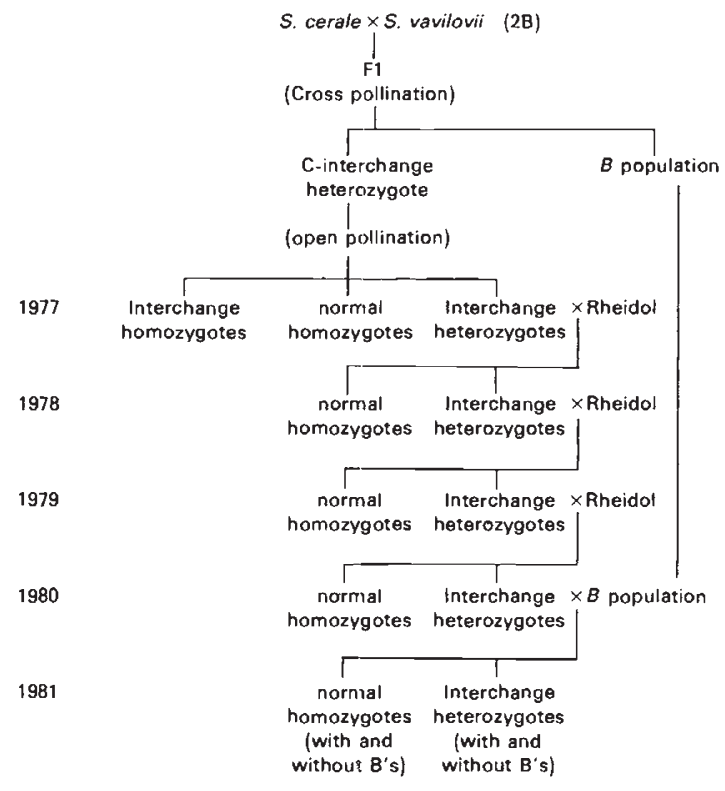

Figure 1 The history of the experimental material.

markedly asymmetrical (see Bailey, Rees and Adena, 1978). From 1971, when the cross was made, until 1977 the stock was maintained by open pollination (see Bailey, Rees and Adena, loc. cit.). The subsequent history of this stock and of the B chromosome stock is given in fig. 1. From the crosses between interchange heterozygotes and plants with B chromosomes in 1980 the following families were derived and scored. 


\begin{tabular}{llll}
\hline & $\begin{array}{l}\text { No. of } \mathrm{B} \\
\text { chromosomes } \\
\text { per plant }\end{array}$ & $\begin{array}{l}\text { No. of plants } \\
\text { Interchange } \\
\text { heterozygotes }\end{array}$ & Normal \\
\hline $\mathrm{A}_{1}$ & 0 & 5 & 5 \\
$\mathrm{~A}_{2}$ & 0 & 5 & 5 \\
$\mathrm{~B}_{1}$ & 2 & 5 & 5 \\
$\mathrm{~B}_{2}$ & 2 & 5 & 5 \\
$\mathrm{C}_{1}$ & 4 & 5 & 5 \\
$\mathrm{C}_{2}$ & 4 & 5 & 5 \\
\hline
\end{tabular}

\section{The comparisons}

In the interchange heterozygotes two groups of chromosomes within the complements are distinguishable at meiosis. The first is comprised of the five bivalents not involved in interchange (the bivalent group). The second includes the four chromosomes implicated in the interchange (the interchange complex), identified by their association as chains or rings of four at meiosis. Since heterozygotes are available without B's, with 2 and 4B's, comparisons could be made to determine whether the B's affect differentially the chiasma frequency of the chromosomes of the bivalent group and those of the interchange complex.

A comparison was made also between normal homozygotes and interchange heterozygotes with and without B's. Interchange homozygotes in this stock are virtually lethal (Bailey, Rees and Adena, loc. cit.) and too few plants were to hand for inclusion in the experiment.

\section{Technical}

Anthers were fixed in Carnoy's fluid and the pollen mother cells stained in aceto-carmine. Chiasma frequency was scored in twenty pollen mother cells in each plant.

\section{RESULT}

\section{(a) The Effect of Interchange upon Chiasma Frequency}

Before describing the effects of B chromosomes it is necessary, as will become evident in a later section, to examine the effect of the interchange itself upon the chiasma frequency within pollen mother cells.

\section{Cell means}

The mean chiasma frequencies in interchange heterozygotes and normal homozygotes without B's are 13.47 and 13.85 respectively (table 1 ). They are not significantly different from one another.

\section{The question of compensation}

That the mean per cell is unaffected by the interchange is surprising. One might expect that the highly asymmetrical nature of this interchange would lead to difficulties with the pairing of the interchange chromosomes at zygotene and, consequently, their capacity to form chiasmata. A possibility to consider is whether the chiasma frequency of the interchange complex itself is, in

Table 1 The mean pollen mother cell chiasma frequencies of plants in families with $0 B, 2 B$ and $4 B$ chromosomes

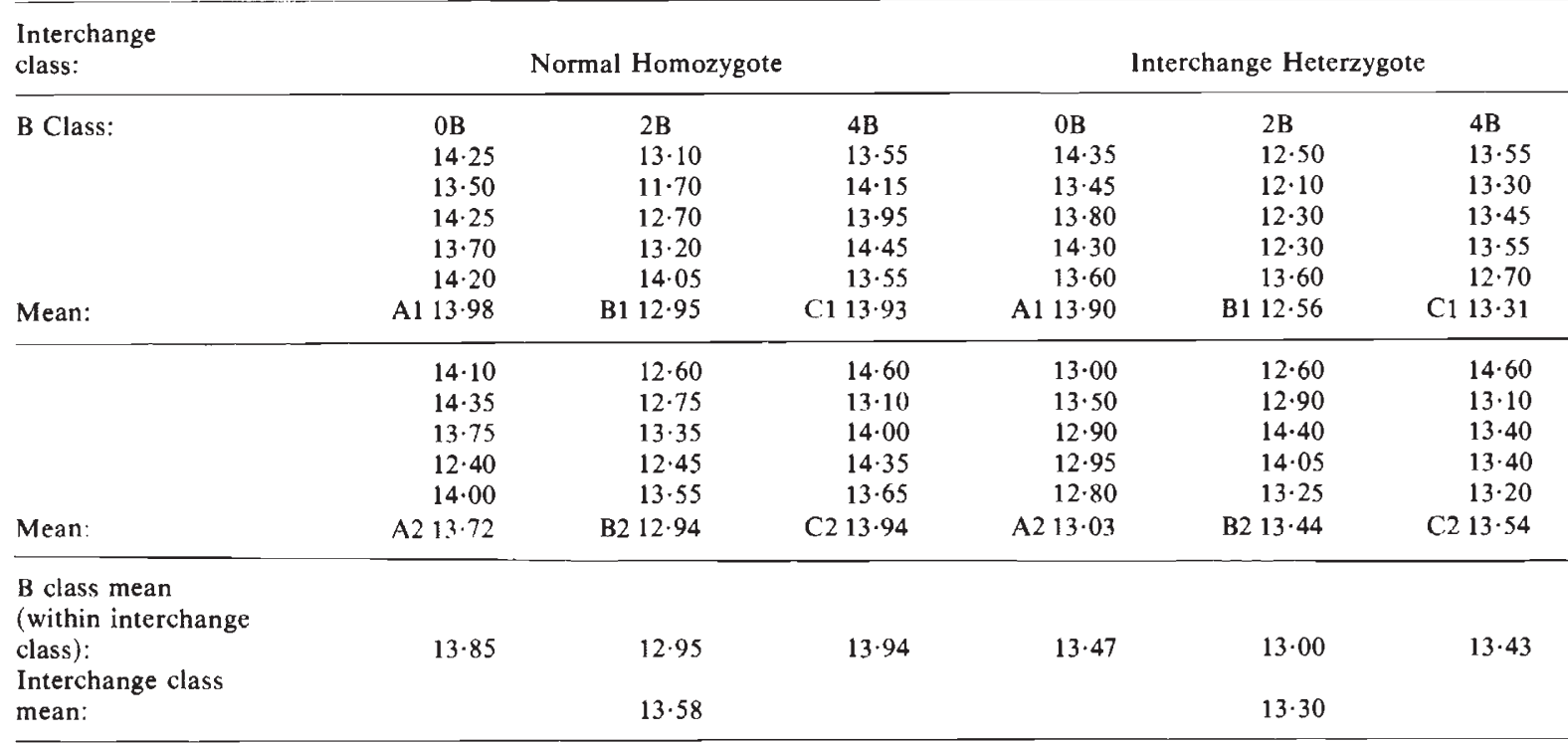




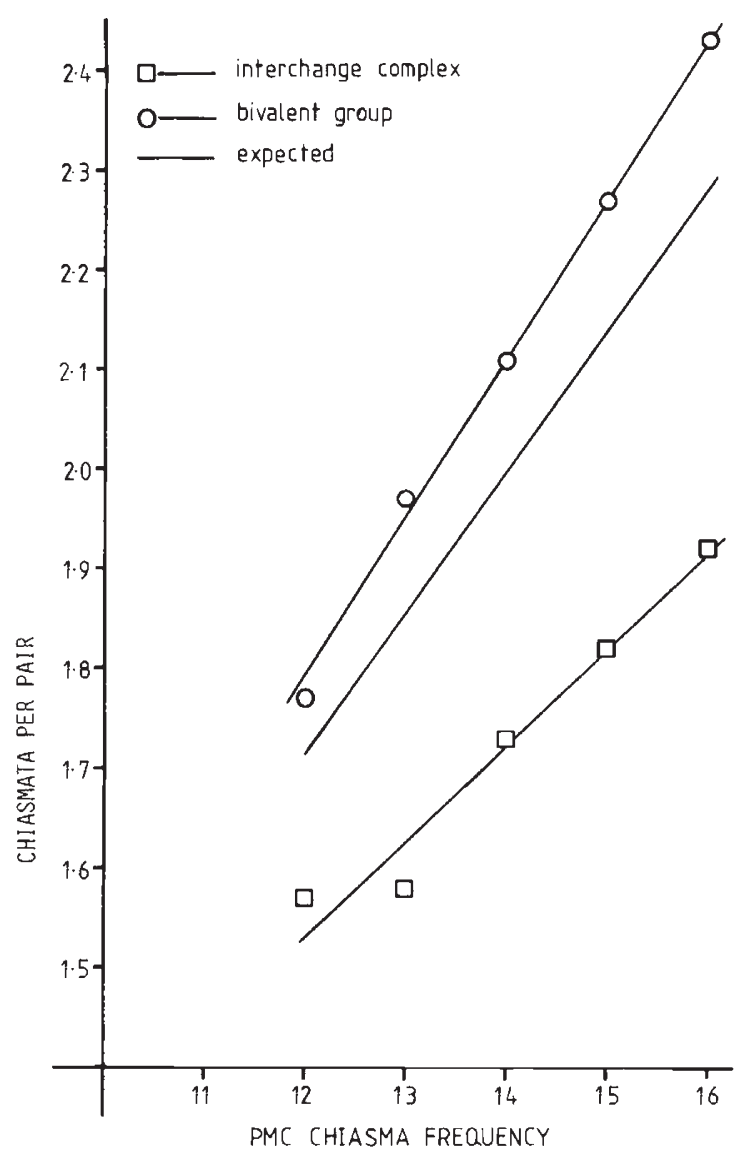

Figure 2 The mean chiasma frequency, "per pair", of chromosomes within the interchange complex and the bivalent group plotted against the pollen mother cell chiasma frequency in $\mathrm{OB}$ interchange heterozygotes. Also, the "expected" frequencies.

fact, reduced but that the mean per cell sustained by a compensatory increase in the bivalent group. Compensatory effects of this kind have been reported in many organisms (Mather and Lamm, 1935; Schultz and Redfield, 1951).

In fig. 2 we have plotted the mean chiasma frequency, "per pair", of the interchange complex and of the bivalent group against the pollen mother cell frequency over the range 12 to 16 in the $\mathrm{OB}$ heterozygotes. We have plotted also the "expected" frequencies, derived on the assumption that the rye chromosomes are of equal length, which is approximately true, and have the same potential to form chiasmata. On these assumptions the expected chiasma frequency, "per pair," of the interchange chromosomes would be similar to that of the bivalent group, namely $2 / 14$ th of the total.

The figure shows the means for the interchange complex to be lower than expected, higher than expected for the bivalent group. The differences are significant $(\mathrm{P}=<0.01)$.

On the face of it the evidence for compensation is strong. It could, however, be questioned on the grounds that the chiasma frequency of the chromosomes involved in the interchange was "inherently" lower than that of the chromosomes of the bivalent group: in other words the chromosomes involved in interchange, even prior to the interchange (that is, as bivalents in the normal homozygote), might have had a lower chiasma frequency than the other five bivalents and, in consequence, lower than the expected values in fig. 2. At meiosis in the normal homozygote we cannot, unfortunately, identify the chromosomes which are involved in the interchange and cannot, as a result, test this possibility directly. It may, however, be tested indirectly, and the case for compensation at the same time confirmed, as follows.

In fig. 3 the mean chiasma frequency, per bivalent, of the two bivalents with the lowest

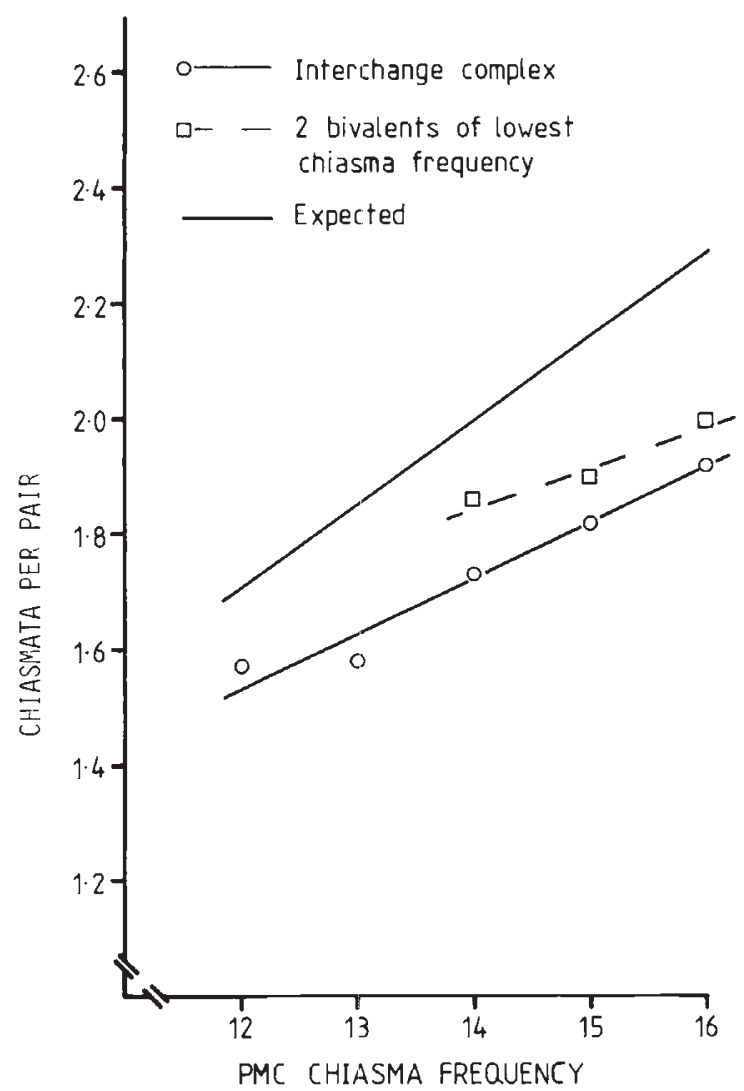

Figure 3 The mean chiasma frequency "per pair" of chromosomes within the interchange complex in $\mathrm{OB}$ interchange heterozygotes and the mean of the two bivalents with the lowest chiasma frequency in normal homozygotes plotted against the pollen mother cell chiasma frequency. 
chiasma frequencies in pollen mother cells of normal homozygotes of families $A_{1}$ and $A_{2}$ are plotted against the pollen mother cell chiasma frequencies. Also plotted are the means, "per pair", of chromosomes of the interchange complex in the interchange heterozygotes in $A_{1}$ and $A_{2}$. The means for the interchange complex are lower than those of the two lowest bivalents. A $t$ test shows that the difference is significant $(P=<0.05)$. It is quite clear, therefore, that interchange is a cause of a reduction in the chiasma frequency of the chromosomes directly involved in interchange. At the same time of course the results confirm, and indeed reinforce, the case for compensation.

It is also important to draw attention in fig. 2 to the difference between the slopes of the regressions of interchange complex and bivalent group means upon the pollen mother cell frequency. That for the interchange complex is significantly lower $(P=<0 \cdot 01)$. As the pollen mother cell chiasma frequency increases the allocation of chiasmata to the interchange complex chromosomes, relative to that of the bivalent group, decreases. As will be explained below, this change in the proportion of the total chiasmata per cell allocated to the two chromosome classes with change in the pollen mother cell frequency bears upon the method of analysis adopted for determining the effects of B's upon the chiasma distribution within the complement.

Leaving aside the question of analytical method this information from the interchange heterozygotes is in itself evidence also for chromosomespecific control, albeit one which is closely dependent on the chiasma frequency of the pollen mother cells. As the latter changes so do the proportions of chiasmata allocated to the interchange complex and the bivalent group. Since the mean chiasma frequency of pollen mother cells in rye is under genotypic control (Rees, 1955) it follows that the allocation of chiasmata in the two different groups of chromosomes must also vary with genotype, if only as a correlated response to change in the pollen mother cell frequency.

\section{(b) The effect of $B$ chromosomes}

(i) Mean frequencies The mean chiasma frequencies per pollen mother cell within all six families are given in table 1 . The means for $2 \mathrm{~B}$ plants are lower than those without B's and with 4B's. The difference is significant $(\mathrm{P}=<0.01)$. There is no significant variation between $\mathrm{OB}$ and $4 \mathrm{~B}$ plants. Evidence from previous work has shown that the effects of B's on chiasma formation are rarely additive (Jones and Rees, 1982). That two B's affect the means, while four B's do not, is not therefore surprising.

(ii) Specific chromosomes. In deciding whether the B chromosomes differentially affect the chiasma frequencies of the interchange complex and the bivalent group chromosomes we need to take account of two kinds of information from the results already described. The first (in 3(b) above) is that the $\mathrm{B}$ chromosomes affect the mean chiasma frequency per pollen mother cell. The second kind of information, in $3(\mathrm{a})$, is that the proportion of chiasmata allocated to the interchange complex and to the bivalent group varies with the pollen mother cell frequency. Because the pollen mother cell frequency itself is affected by B's the possibility then arises, as we have explained, that a change in the proportions of chiasmata allocated to the two chromosome classes in the presence of B's might follow simply as a consequence of a change in the pollen mother cell chiasma frequency. The complication raised by this possibility may readily be overcome, however, by making comparisons between pollen mother cells of similar chiasma frequency, in plants with and without B chromosomes.

In fig. 4 the mean number of chiasmata in the interchange complex is plotted against the chiasma frequency of pollen mother cells from interchange heterozygotes with $\mathrm{OB}, 2 \mathrm{~B}$ and $4 \mathrm{~B}$ chromosomes. In pollen mother cells of similar chiasma frequency there is no significant difference between the number of chiasmata allocated to the interchange complex in $0 \mathrm{~B}$ and $4 \mathrm{~B}$ plants. With 2B's, in contrast, the chiasma frequency of the interchange complex, overall, is higher than for $0 \mathrm{~B}$ and $4 \mathrm{~B}$ plants. (This means, of course, that the allocation of chiasmata to the bivalent group is lower). The difference between the means is significant $(P=<0.02)$ as shown by a regression analysis of variance embracing the chiasma range 12 to 15 , in which all $\mathrm{B}$ classes are represented. Figure 4 also shows that in $2 \mathrm{~B}$ plants there is a disproportionate increase in the chiasma frequency of the interchange complex with increase in the pollen mother cell frequency. This is confirmed by the difference between the slopes of the regression lines for $2 \mathrm{~B}$ plants, on the one hand, and the slopes for $0 \mathrm{~B}$ and $4 \mathrm{~B}$ plants on the other $(\mathrm{P}=<0.001)$.

From the results it is clear that in cells with similar chiasma frequencies the B chromosomes, at a certain dosage, viz. $2 \mathrm{~B}$, affect differentially the distribution of chiasmata to particular chromosomes within the complement, namely the chromosomes of the interchange complex and those of the 


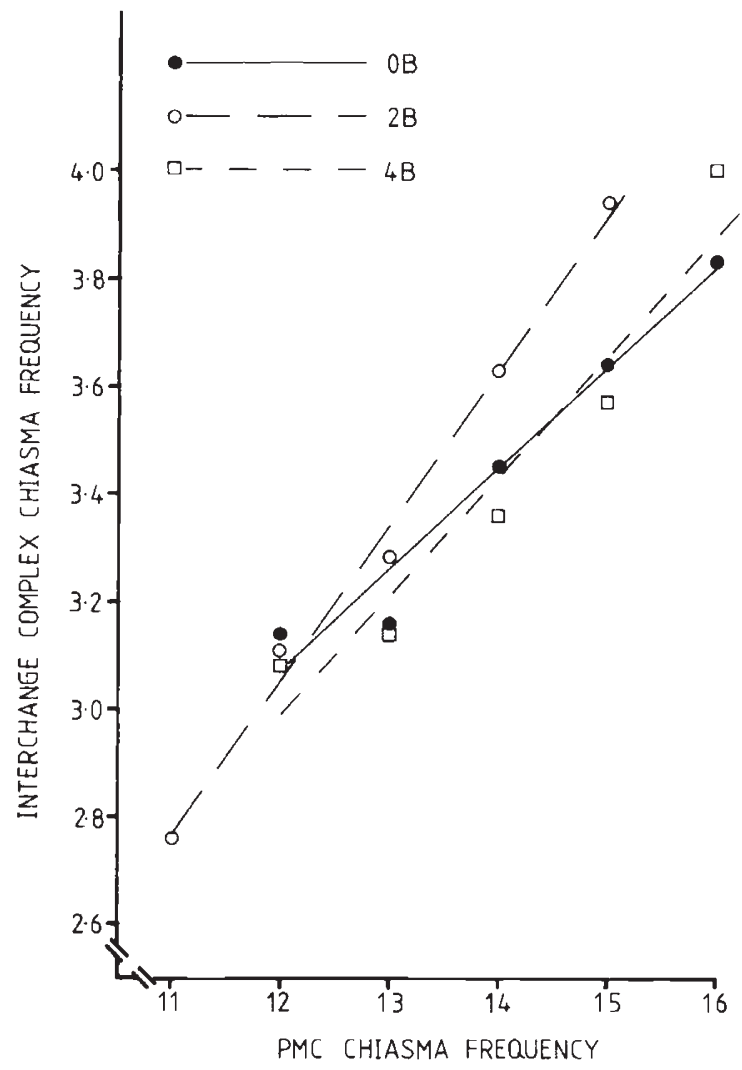

Figure 4 The mean chiasma frequency of the interchange complex in $0 \mathrm{~B}, 2 \mathrm{~B}$ and $4 \mathrm{~B}$ interchange heterozygotes plotted against the pollen mother cell chiasma frequency.

bivalent group. The analysis shows, at the same time, that the magnitude of the effect of B's varies in relation to the total pollen mother cell chiasma frequency. This latter effect is made particularly clear in fig. 5 in which the proportion of chiasmata allocated to the interchange complex in $0 \mathrm{~B}$ and $2 \mathrm{~B}$ plants is plotted against the pollen mother cell frequency. With increase in the pollen mother cell chiasma frequency in $2 \mathrm{~B}$ plants the proportion allocated to the interchange complex increases, at the expense of course of the bivalent group. In $0 \mathrm{~B}$ plants the proportion decreases. A joint regression analysis of variance, following angular transformation, confirms a highly significant heterogeneity between the regression slopes $(\mathrm{P}=<0 \cdot 01)$.

\section{DISCUSSION}

The interchange proved to be an effective means for distinguishing particular chromosomes within the rye complement. At the same time the choice of an interchange as a "chromosome marker" was,

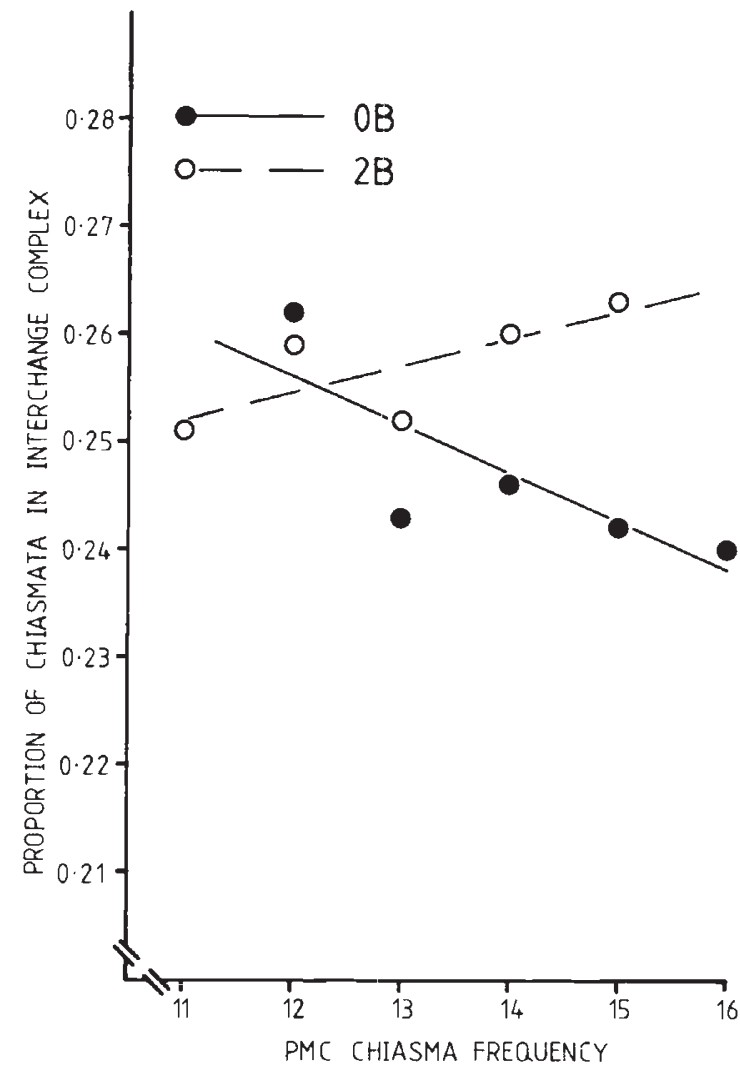

Figure 5 The mean proportion of the total chiasmata in pollen mother cell allocated to the interchange complex in $0 \mathrm{~B}$ and 2B interchange heterozygotes plotted against the pollen mother cell chiasma frequency.

in itself, rewarding in generating information about the consequences of structural change upon the distribution of chiasmata within a complement. This information is given in the summary.

The chief objective of the investigation was to seek evidence for genotypic control, affecting chiasma frequency, over specific chromosomes within a complement. The results provide conclusive evidence to this effect. Moreover, the results show that, to a very large degree, different numbers of chiasmata may be allocated to different chromosomes within the complement independently of any change in the total for the complement as a whole. In a recent paper we have demonstrated, in a somatic hybrid in Petunia (White and Rees, 1985), how the chiasma frequencies of different genomes within the hybrid complement are specifically and separately determined. Considered together, and in conjunction also with the evidence from Parker (1975) and from Tease and Jones (1976), the results are of significance in the following context. The amount of recombination within 
particular gene complexes within a complement are genotypically determined. It follows that the spectrum of genetic variability released to the gametes is subject to adjustment determined by heredity. For particular gene complexes it may be restricted, for others facilitated. In either case the consequences must profoundly affect the fate of the resulting progenies exposed to selection.

\section{SUMMARY}

An interchange was used as a marker for identifying particular chromosomes within the rye complement. In pollen mother cells the four chromosomes involved in interchange have a lower chiasma frequency in the interchange heterozygote than in the normal homozygote. In the interchange heterozygote there is, however, a compensatory increase in the chiasma frequency of the five bivalents not involved in interchange, with the result that the mean chiasma frequency of pollen mother cells is maintained at the same level as that of the normal homozygote.

Comparisons were made between the chiasma frequencies of both interchange heterozygotes and normal homozygotes with varying numbers of $B$ chromosomes; 0,2 and 4 . The mean chiasma frequency of heterozygotes and homozygotes was higher in plants with $2 \mathrm{~B}$ 's than with $0 \mathrm{~B}$ and $4 \mathrm{~B}$ 's. In the interchange heterozygotes the $2 \mathrm{~B}$ 's affected differentially the chiasma frequency of the chromosomes involved in interchange and the chromosomes not involved in interchange. In general the proportion of the total chiasmata in pollen mother cells allocated to the interchange chromosomes, at the expense of chromosomes not involved in interchange, is higher in $2 \mathrm{~B}$ than in $\mathrm{OB}$ and $4 \mathrm{~B}$ plants. The magnitude of this differential effect increases with increase in the chiasma frequency of pollen mother cells. On the basis of this evidence it is concluded that control exerted by the genotype, in this case by B chromosomes, may determine the amount of recombination at meiosis within particular gene complexes within the chromosome complement and, in consequence, the pattern of variability released to the gametes and progenies.

\section{REFERENCES}

BAILLYY, R. J., REES, H. AND ADENA, M. A. 1978. Interchange heterozygosity and selection in rye. Heredity, 41, 1-12.

JONES, R. N. AND REES, H. 1967. Genotypic control of chromosome behaviour in rye. XI. The influence of $B$ chromosomes on meiosis. Heredity, 22, 333-347.

JONES, R. N. AND REES, H. 1982 B Chromosomes. Academic Press.

MATHER, K. AND LAMM, R. 1935. The negative correlation of chiasma frequencies. Hereditas, 20, 65-70.

PARKER, J. S. 1975. Chromosome-specific control of chiasma formation. Chromosoma, 49, 391-406.

REFS, H. 1955. Genotypic control of chromosome behaviour in rye. 1. Inbred Lines. Heredity, 9, 93-116.

SCHULTZ, J. AND REDFIELD, H. 1951. Interchromosomal effects on crossing-over in Drosophila. Cold Spring Harbor Symp. Quant. Biol., 16, 175.

TEASE, C. AND JONES, G. H. 1976. Chromosome-specific control of chiasma formation in Crepis capillaris. Chromosoma, 57, 33-49.

WHITE, JULIA AND REES, H. 1985. The chromosome cytology of a somatic hybrid Petunia. Heredity, 55, 53-59. 